Classification

Physics Abstracts

05.20. $-\mathrm{y}-62.60 \mathrm{Mk}-77.50 .+\mathrm{p}-72.90 .+\mathrm{y}$

\title{
Rupture of central-force lattices
}

\author{
A. Hansen $\left({ }^{1}\right)$, S. Roux $\left({ }^{2}\right)$ and H. J. Herrmann $\left({ }^{3}\right)$ \\ (1) Institut für Theoretische Physik, Universität zu Köln, D-5000 Köln 41 F.R.G. \\ (2) Laboratoire de Physique de la Matière Hétérogène, URA 857, Ecole Supérieure de Physique \\ et Chimie Industrielles, 10 rue Vauquelin, F-75231 Paris Cedex 05, France \\ (3) Service de Physique Théorique, C.E.N. Saclay, F-91191 Gif-sur-Yvette Cedex, France
}

(Reçu le 10 juin 1988, revisé le 5 décembre 1988, accepté le 7 décembre 1988)

\begin{abstract}
Résumé. - Nous étudions numériquement la rupture de treillis réticulés élastiques dont les liens sont élastiques-fragiles. Tous les liens ont une même raideur, mais une force critique de rupture distribuée aléatoirement de façon uniforme entre 0 et 1 . Nous analysons la caractéristique globale moyenne force-déplacement ainsi que d'autres paramètres physiques en fonction de la taille du système. Nous présentons des relations d'échelle liant ces quantités. Finalement, nous montrons que la distribution des forces est multifractale au seuil ultime de rupture du réseau.

Abstract. - We study numerically the rupture of elastic lattices consisting of fragile elastic springs which can freely rotate around nodes. All bonds are given an identical force constant but the threshold force for which they break is randomly attributed to each bond according to a uniform probability distribution between 0 and 1 . We analyse the overall force-displacement characteristics of the lattices and other physical parameters as function of lattice size. Various scaling relations are found. Finally, we found evidence for a multifractal distribution of forces at the ultimate stage of the rupture.
\end{abstract}

\section{Introduction.}

An old subject in engineering sciences has been the study of fracture processes in heterogeneous materials [1]. However, due to the complex interplay of the "quenched" disorder (of the elastic failure characteristics of the medium) and of the increasingly large distribution of local stresses as the rupture proceeds through the medium, very few theoretical results have been obtained. In addition to this, numerical simulations of this problem are in general very « heavy».

For these reasons, a natural strategy is to simplify the complex realistic cases in order to capture first the essential properties of fracture which are independent - or at least only weakly dependent - on the underlying microscopic (if the rupture proceeds on an atomic scale) or mesoscopic scale (when the heterogenities are large compared to the atomic scale, such as in the rupture of concrete). To an increasing degree, the methods of statistical mechanics have been found useful in the study of the scaling properties of real phenomena such as aggregation processes, etc. We attempt here such an approach to the problem of rupture. 
We considered specifically a two-dimensional lattice model, where the local elastic moduli are uniform and, finally, where disorder is introduced once at the beginning in the distribution of failure thresholds. In this way, all the heterogenities of the material are captured in only one quantity, thus making it possible to investigate which features of the heterogenities are important during various stages of the rupture process.

The fuse problem [2-5] is one obvious simplification which has very often been studied since it is the scalar equivalent of elastic rupture. It is now well-known that, in some respect, vector and scalar problems differ significantly even in the scaling relations they obey, for instance, in percolation [6]. From a practical point of view it is very important to understand which features of the real rupture processes can be captured by using a scalar model only, for the obvious reason that scalar problems are eomputerwise much less time consuming than vector ones - besides being much simpler to work with theoretically. For this reason, we followed the very same procedure and gathered the same information for three parallel cases [7] :

- an elastic central-force lattice which will be described in this article ;

- a beam lattice where each bond has a non-zero rigidity for axial and transverse force, and for bending [8] ;

- a fuse lattice defining the corresponding scalar problem [9].

The reason for treating separately the central-force model and an angular elasticity model as the beam model, is that the concept of rigidity in the central-force model is non-local [10], making it possibly very different from the other models where the local elastic rigidity is determined solely by the local connectivity properties of the lattice. On the other hand, in the central-force model there are no free parameters since all the disorder is in the breaking strengths as opposed to the angular elasticity case.

Within the framework of our model, there are still many possibilities to introduce disorder. One natural way is to consider a percolation-type disorder, i.e. a finite fraction of bonds are removed at random, whereas the remaining ones have identical elastic properties, and the same failure threshold. This latter case has been considered in the past both for the fuse problem [3] and the central-force case [11]. We consider here a different kind of disorder, by introducing a continuous distribution of failure strength. This lead to very different results than previously reported ones. We discuss the comparison of these two types of disorder later in this paper.

\section{Model.}

We consider a triangular lattice where each bond consists of a linear Hookean spring free to rotate around its endpoints at the nodes of the lattice. The elastic constant of these springs is fixed to be unity by definition. Each bond is ideally brittle and breaks (i.e. its elastic constant becomes zero) whenever the force $\mathrm{f}$ it is subjected to exceeds a positive limit $f_{+}$(if the bond is stetched) or a negative limit $f_{-}$(if the bond is compressed). We consider here the case of a symmetric rupture criterion $f_{+}=f_{-}=f_{\mathrm{c}}$. Contrary to the elastic modulus, the threshold $f_{\mathrm{c}}$ for each bond is randomly picked, according to a probability density that we choose to be uniform between 0 and 1 . We assume that the bonds are very brittle, i.e. their deformation at rupture is small compared to their length at rest. This allow us to treat the problem within the limit of small deformation, i.e. to deal with a linear problem. This linearity property will be used all along the paper. Since in the linearized equations there does not exist any length scale, we will use a scale for force given by the distribution of breaking threshold, and a scale for displacement given by the previous in setting arbitrarily the elastic modulus to unity. This length scale with which we measure the deformation of the lattice has no direct relation with 
the lattice size. (Our small deformation assumption means that the lattice spacing expressed in our length units is large compared to 1.)

The boundary conditions are periodic in the horizontal direction, and the top and bottom rows are attached to two rigid bars at which we impose a given displacement : either a shear or a compression.

The numerical procedure used is the following :

- we start with a regular lattice. For an imposed unit displacement of the rigid bars, we know the force distribution in the lattice. Let $f_{i}$ be the force to which the $i$-th bond is subjected to. Let us now imagine that the displacement of the rigid bars is $\Lambda$ instead of 1 , then the force at bond $j$ is $\Lambda f_{j}$. If the displacement $\Lambda$ is increased from 0 , the bond $j$ will break when

$$
\Lambda\left|f_{j}\right|=f_{j \mathrm{c}} .
$$

We thus compute for each bond $j$ :

$$
\Lambda_{j}=f_{j \mathrm{c}} /\left|f_{j}\right|
$$

and break the bond for which $\Lambda_{j}$ has the smallest value $\Lambda_{c}=\min _{j}\left(\Lambda_{j}\right)$ which corresponds to the overall lattice displacement for which a first bond will break. After the bond $j$ is broken, the new force distribution is computed. The new value of $\Lambda_{c}$ is found, and the computation is repeated until the entire lattice has a zero elastic modulus.

The complete « history » of the rupture of the lattice is given by the sequence of the displacements $\Lambda_{c}(n)$, of the total external forces $F(n)$, of the elastic modulus $Y(n)=$ $F(n) / \Lambda_{c}(n)$, of local forces $f(n)$ for a unit external displacement of the bond that will break... indexed by $n$, the number of already broken bonds.

Now, in order to obtain an average behavior, one has to take a mean over many samples. In each sample, some controllable quantity has to be kept constant in order to be able, in a meaningful way, to compare the samples. In the language of statistical mechanics, an « ensemble » has to be chosen [12]. This question is not unimportant since it might very well turn out that the resulting average behaviors differ, according to which quantity is kept constant in comparing the different samples.

In this article (and in its companions [7-9]), we choose an « history » average. Any quantity $X(n)$ is averaged over different lattices keeping $n$ constant, and therefore, restricting the average to the lattices that are not yet torn apart at this stage of the rupture. One should note that the number of samples the quantity is averaged over will decrease with increasing $n$. We generated $100004 \times 4,10008 \times 8,30016 \times 16$ and $3024 \times 24$ lattices.

The algorithm we used to solve the force distribution on the lattices was a conjugate gradient relaxation method without Fourier acceleration [13] - for the lattice sizes we considered, Fourier acceleration turned out to be unefficient.

\section{Results.}

Figure 1 shows the total displacement, $\left\langle\Lambda_{c}(n)\right\rangle$, (elongation) at rupture as a function of the number, $n$, of bonds broken for different lattice sizes $L$. A striking feature of these graphs, is that they clearly reveal a linear relation between these two quantities for $n$ up to 100 forL $=16$ and $n=200$ for $L=24$. After this linear regime, the displacement increases drastically. On these graphs, we also draw the straight line given by:

$$
\left\langle\Lambda_{c}(n)\right\rangle=(1 / \sqrt{3}) n / L
$$

that fits the first regime very nicely. 


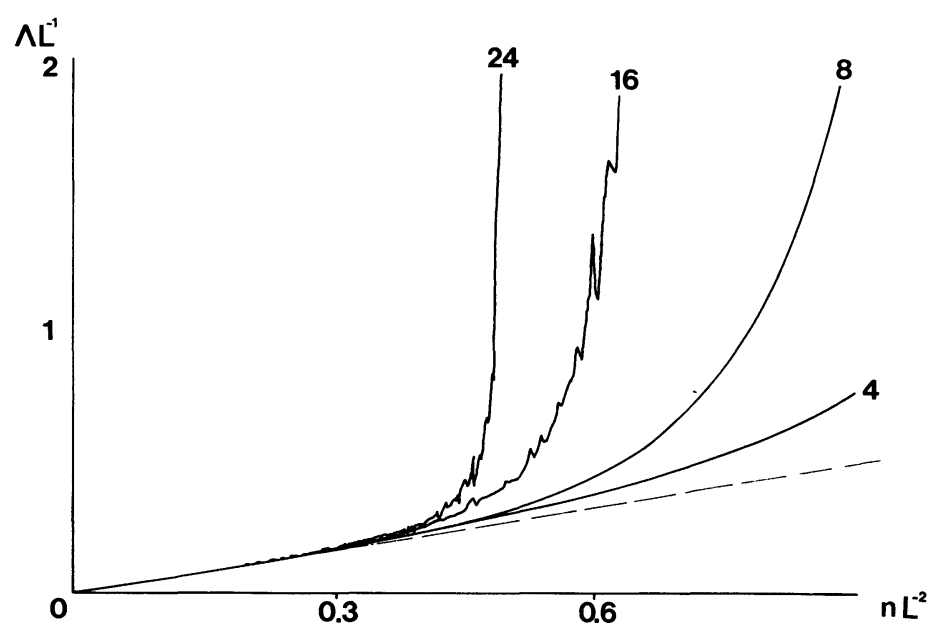

Fig. 1. - Evolution of the overall lattice displacement per unit length $\Lambda L^{-1}$ at rupture as a function of $n L^{-2}$, the number of bonds already broken divided by $L^{2}$. This displacement is averaged over different lattices with the same $n$. The four curves refer to four different lattice size $4,8,1$ and 24 . The straight line is the linear relation of equation (3) in the text.

We can understand this relation by the following crude, mean-field type, argument : let us assume that the strain is uniformly distributed in the lattice (as if it were intact). Since the imposed displacement is an elongation, each bond parallel to the top and bottom rigid bars will carry no force, due to the periodic boundary conditions. On the contrary, the bonds in the other two directions will be elongated by an amount $\varepsilon=(\sqrt{3} / 2) \Lambda / L$. For a given $\Lambda$, the bonds that have been broken are those whose strain rupture threshold is less than $\varepsilon$. In the case of a uniform distribution of rupture thresholds between 0 and 1 , the number of these bonds carrying a strain less than $\varepsilon$ is given by the equation

$$
n=2 L^{2} \varepsilon,
$$

resulting in equation (3).

The basic assumption of this argument is certainly very rough; however, it gives an unexpectedly good result (see Fig. 1). Let us note that the mean-field prediction of the elastic modulus of the randomly depleted central-force triangular lattice [14] also works remarkably well, almost up to the percolation threshold.

Figure 2 gives the force needed to break $n$ bonds for the $L=16$ and 24 lattices. In this case, we performed a parabolic fit of the data according to :

$$
\left\langle F_{\mathrm{c}}(n)\right\rangle=\left\langle\Lambda_{\mathrm{c}}(n)\right\rangle\left(1-\alpha n / L^{2}\right)
$$

or, equivalently, we searched for the first linear correction to the elastic modulus, $Y(n)=\langle F(n)\rangle /\left\langle\Lambda_{c}(n)\right\rangle$. Figure 2 displays these best fits which are quite good up to the apex of the parabola $(n \approx 90$ for $L=16$ and $n \approx 160$ for $L=24)$. They were obtained for $\alpha=1.0, \alpha=1.25, \alpha=1.5$ and $\alpha=1.65$, respectively for $L=4,8,16$ and 24 . It turned out that it was not possible to find a constant value of the parameter $\alpha$ that could fit all sizes equally well. On the contrary, $\alpha$ tends to increase with $L$. We will come back to this point later. We also note that if the rupture of bonds would be done completely at random (for instance if the rupture thresholds would dominate the ratios of Eq. (2)), then the parameter 


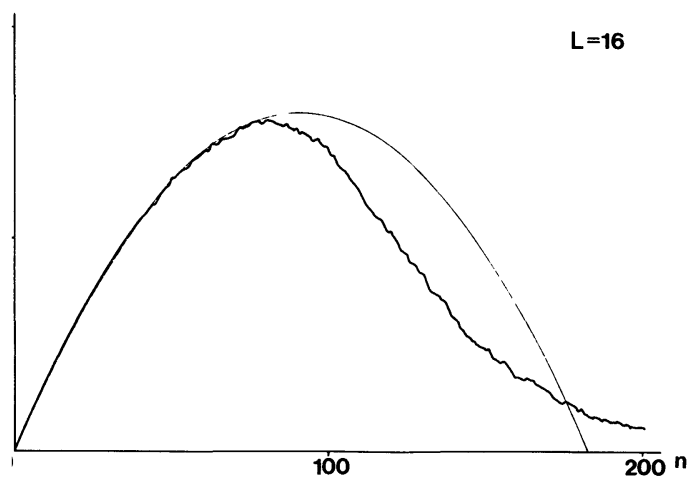

a)

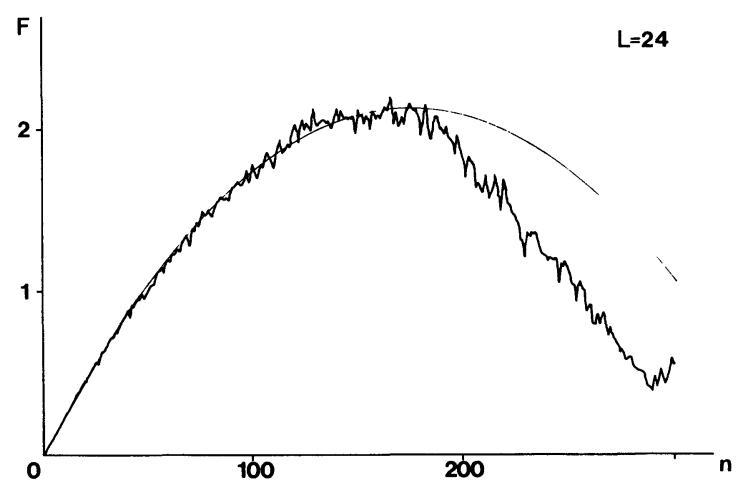

b)

Fig. 2. - Evolution of the overall force imposed to the lattice at rupture as a function of $n$, the number of bonds already broken. Figure $2 a$ refers to lattice size 16 (300 samples), and $2 b$ to size 24 (100 samples). In each graph we have plotted the best parabolic fit of the first data points. (See Eq. (5) in the text.)

$\alpha$ would have been given by the mean-field behavior encountered for random depletion, i.e. $\alpha=1$ [14].

We also recorded the average elastic modulus $\langle Y(n)\rangle$ shown in figure 3 . This graph reveals that a linear approximation for the dependence of $\langle Y(n)\rangle$ on $n$ is clearly insufficient to account for its evolution.

In order to see which part of the ratio (r.h.s. of Eq. (2)) controls the rupture, we also recorded the stress, $f(n)$, on the bond that was about to break, for a unit displacement. Figure 4 displays the evolution of this quantity. We can see clearly that there exists a first regime where this value is noisy, but constant on the average, and a second one where this force drops to zero. It had been suggested [15] that a first regime of fracture is controlled only

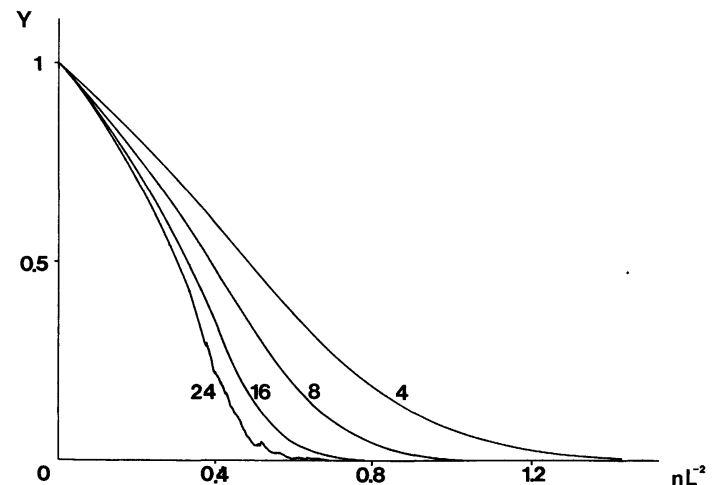

Fig. 3 .

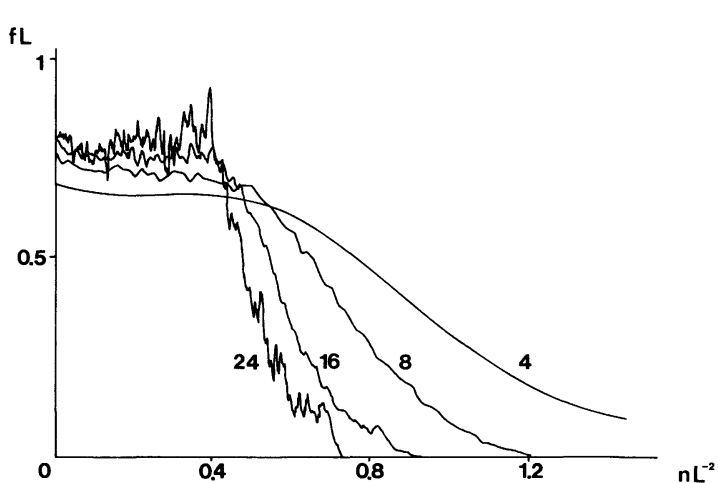

Fig. 4.

Fig. 3. - Elastic modulus versus number of bonds broken divided by $L^{2}$ for the four lattice sizes considered.

Fig. 4. - Force acting on the bond that is to be broken when a unit displacement per unit length is imposed onto the lattice. The first regime is characterized by a constant, though noisy, force which decreases slowly with the lattice size. 
by the disorder of thresholds. In other words, at the beginning of the rupture process we would expect that the stresses onto the bonds of the lattice are more or less the same, whereas the strengths of the bonds are widely distributed, therefore, through equation (2), the breaking of bonds and the force $f(n)$ should be uncorrelated. This expectation is in agreement with the fact that $f(n)$ is independent of $n$. We also notice that $f(n)$ decreases when the lattice size increases and from the previous analysis, we get a $1 / L$ dependence which is approximately verified (Fig. 4).

The approach followed up to now, i.e. relating the evolution of the observed quantities with the number of bonds broken, is clearly unable to give a general description of the process, independent of lattice sizes. Increasing the order of the expansion in $n$, would certainly give better firts for a given size, however, without any knowledge of the evolution of the coefficients of the expansion (e.g. like $\alpha$ ) this procedure is useless. We thus turn to another approach that takes into account these size effects more properly.

\section{Rescaling.}

We now try to find a simple relation between the different quantities reported up to now on the lattice size. In this spirit, we propose the following Anzatz :

$$
\left\langle F_{\mathrm{c}}(n)\right\rangle=L^{\beta} \Phi\left(\left\langle\Lambda_{\mathrm{c}}(n)\right\rangle L^{-\gamma}\right)
$$

where $\beta$ and $\gamma$ are unknown exponents and $\Phi$ is a universal function independent of the system size. In physical terms, this scaling relation defines some reduced variables, here $\left\langle F_{\mathrm{c}}(n)\right\rangle L^{-\beta}$ and $\left\langle\Lambda_{\mathrm{c}}(n)\right\rangle L^{-\gamma}$, which are related through a function which is independent of lattice size. The values of $\beta$ and $\gamma$ are determined through the best collapse of the data obtained for different sizes. Such a fit is shown in figure 5 for $\beta=\gamma=3 / 4$. Other trial functions were also used, but the simple form of equation (6) fitted the data remarkably well in the first part of the curve (a little after the apex of the breaking force) if we disregard the data of size 4 which is obviously too small to give any reliable estimates.

Using the property (seen previously) that the slope in the force-displacement relation in the immediate vicinity of 0 is 1 (the elastic modulus is close to 1 at the beginning of the rupture),

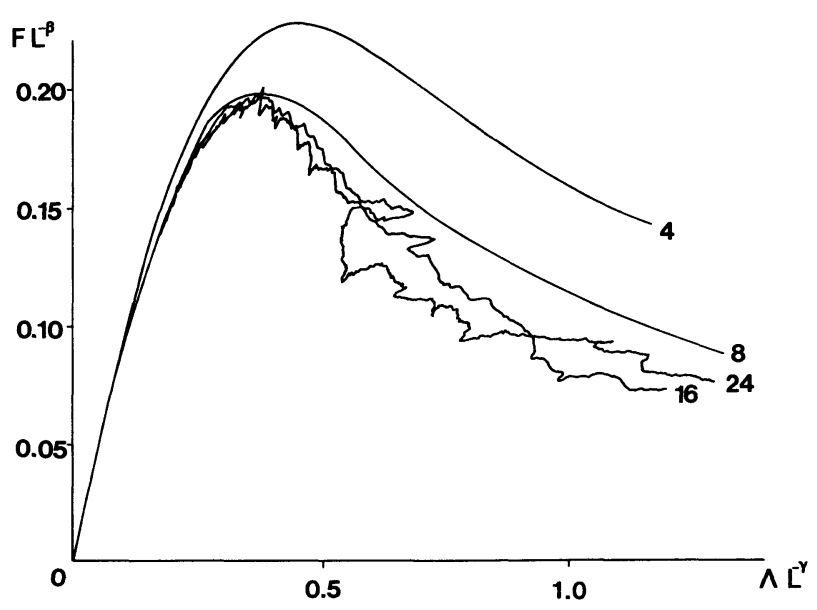

Fig. 5. - Rescaled force-displacement relation according to equation (6) with $\beta=\gamma=3 / 4$, for different sizes $4,8,16$ and 24 . 
we obtain directly that $\beta=\gamma$, in agreement with the result obtained above through the fitting procedure.

If now we rewrite the development of $\left\langle\Lambda_{c}(n)\right\rangle$ and $\left\langle F_{c}(n)\right\rangle$ respectively to the first and second order in $n$ as a function $F_{\mathrm{c}}\left(\Lambda_{\mathrm{c}}\right)$, we obtain using equations (3) and (5)

$$
F_{\mathrm{c}}\left(\Lambda_{\mathrm{c}}\right)=\Lambda_{\mathrm{c}}\left(1-\alpha \sqrt{3} / L \Lambda_{\mathrm{c}}\right)
$$

Using the scaling relation equation (6) yields

$$
\alpha \propto L^{1-\beta}
$$

where we have used $\beta=\gamma$.

The smooth increase of $\alpha$ with $L$ that was noted previously is consistent with this relation since $1-\beta=0.25$ is a very small exponent. (Fig. 6 shows it in a more quantitative way.)

The number of bonds broken at the maximum breaking force can also be obtained accordingly since we have seen before that the linear $\left(\Lambda_{c}\right)$ and the quadratic $\left(F_{c}\right)$ approximations were valid at least up to the apex of the force. Differentiating equation (7) gives :

$$
\Lambda_{c \max }=L /(2 \sqrt{3} \alpha) \propto L^{\beta}
$$

the number of bonds broken at this point should vary as (see Eq. (3))

$$
n_{\max } \propto L^{\beta+1}
$$

and the maximum force $F_{\max }$ should scale as :

$$
F_{\max } \propto L^{\beta}
$$

We have plotted the different quantities $L / \alpha, \Lambda_{c \max }, n_{\max } / L$ and $F_{\max }$ as a function of the lattice size on a log-log plot in figure 6. All those quantities should follow a power-law with the exponent $\beta$. We thus get four determinations of this exponent that should be compared to the $3 / 4$ obtained from the global force-displacement characteristic. These estimates of $\beta$ read : $0.74,0.69,0.68,0.77$ respectively for the four quantities mentioned above. We see that these determinations of the exponent $\beta$ are consistent, and give in addition a rough estimate of the uncertainty on the value of $\beta$.

We also note that a similar rescaling is obtained in other models considered in reference [8] for an elastic lattice with angular elasticity, and in reference [9] for a fuse model. A surprising observation is that the value of $\beta$ seems identical in all three models [7].

Another interesting output of the computation is the total number of bonds broken when the lattices get a zero elastic modulus. This number $n_{\text {tot }}$ is to be distinguished from $n_{\max }$ which refers to the number of bonds broken at the apex of the force-displacement curve. However both numbers appear to follow a power law with $L$. In figure 6 we have reported the value of $n_{\text {tot }} / L$. The apparent slope is about 0.6 , resulting in $n_{\text {tot }} \propto L^{1.6}$, somewhat smaller than, but not inconsistent with, the corresponding law for $n_{\max }(1+\beta)$. In the other cases, references $[8,9]$, where larger sizes were investigated, $n_{\text {tot }}$ and $n_{\max }$ seemed to follow the same behavior. We also note that, for the central-force case considered here, the final stage of rupture is not necessarily a complete partition of the lattice into two separate pieces : the rupture stops when the elastic modulus of the lattice drops to zero, even if the system is still connected. This is a particularity of the central-force model, that is not shared by the other models where a non-zero rigidity (or conductivity) is in one to one correspondance with connectivity. 


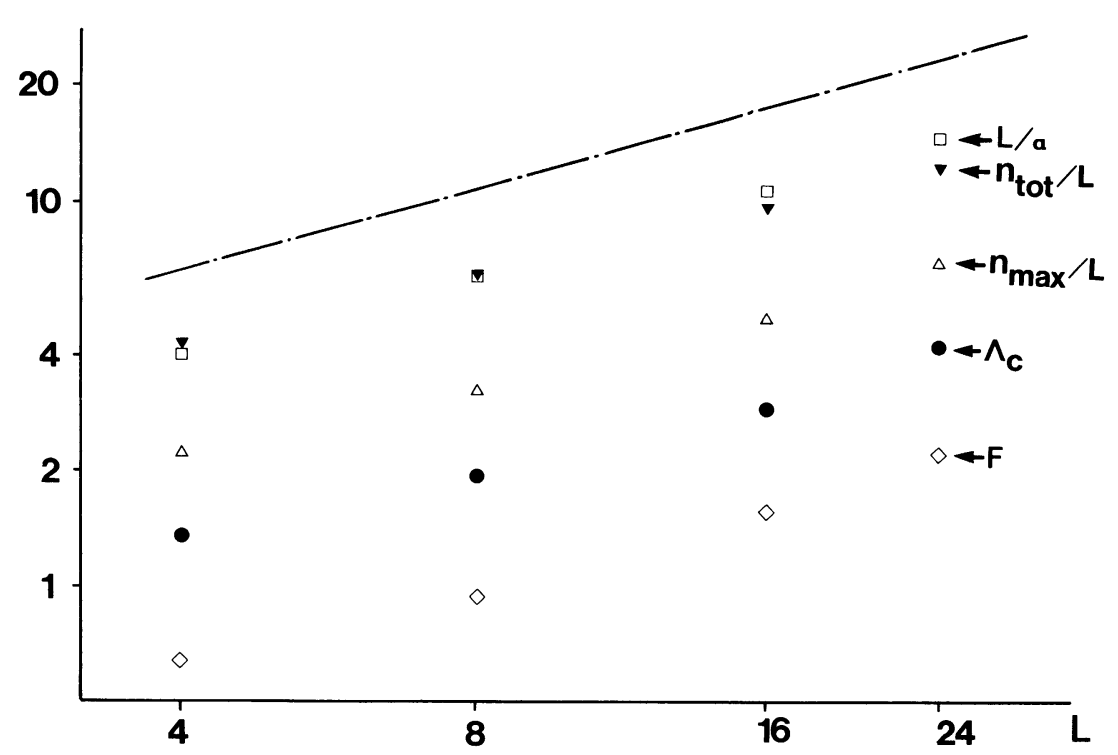

Fig. 6. - Log-log plot of $L / \alpha, \Lambda_{\mathrm{c} \max }, n_{\max } / L, n_{\mathrm{tot}} / L$ and $F_{\max }$ as a function of the lattice size $L$. All those quantities should scale with the same exponent $\beta$. The slope that would result from $\beta=3 / 4$ is shown as a straight line on the graph.

We would like to emphasize the fact that one parameter of the rescaling, e.g. $\beta$, has been determined exclusively from numerical data. It certainly deserves an explanation that we do not have at present. Theoretical approaches of this problem as references [3, 5] would rather suggest an estimate of $\beta$ of order 0 (logarithmic term) or 1 , which are both incompatible with our data. As a result of the strong generality of the results obtained for our models of rupture, a conjecture has been made [7] that relates the exponent of the scaling of $n_{\max }$ and $n_{\text {tot }}$ versus $L$ with the fractal dimension of «diffusion-limited aggregation ». This conjecture however deserves further studies that are being performed.

Finally, we also record the complete histogram $N(f, L)$ of the logarithm of the force distribution, just before the last bond breaks. In order to check the eventual « multifractal » [16] nature of this distribution we plot in figure $7, \log (N(f, L)) / \log (L)$ versus $\log (f) /$ $\log (L)$. If the distribution is multifractal, this plot should tend to be size-independent for large enough lattice and converge to the so-called « $f(x)$ » spectrum (we use this notation as the «standard » one, however $\alpha$ has nothing to do with the coefficient discussed previously), thus containing all available information on the scaling behavior of the average force distribution inside the lattices at the breaking point. Indeed figure 7 seems to suggest this property, although certainly the sizes considered here are too small to lead to a definitive conclusion in this regard.

Another equivalent way of obtaining the multifractal spectrum $f(\alpha)$, is through the scaling of the moments of the force distribution. Let us define $M_{q}=\sum_{i}\left|f_{i}\right|^{q}$ where the sum runs over all bonds carrying a non-zero force. These moments scale with $L$ according to $M_{q} \propto$ $L^{-p(q)}$. In particular, $M_{0}$ is the mass of the force-carrying part of the lattice. Since the density of broken bonds $n_{\text {tot }} / L^{2}$ represents a vanishingly small fraction of the whole lattice, we expect $p(0)=-2$. This is indeed what we observed $(p(0)=2.01$ from the direct measurement). Figure 8 shows the evolution of the reduced moments $m_{q}=\left(M_{q} / M_{0}\right)^{1 / q}$ as a function of 


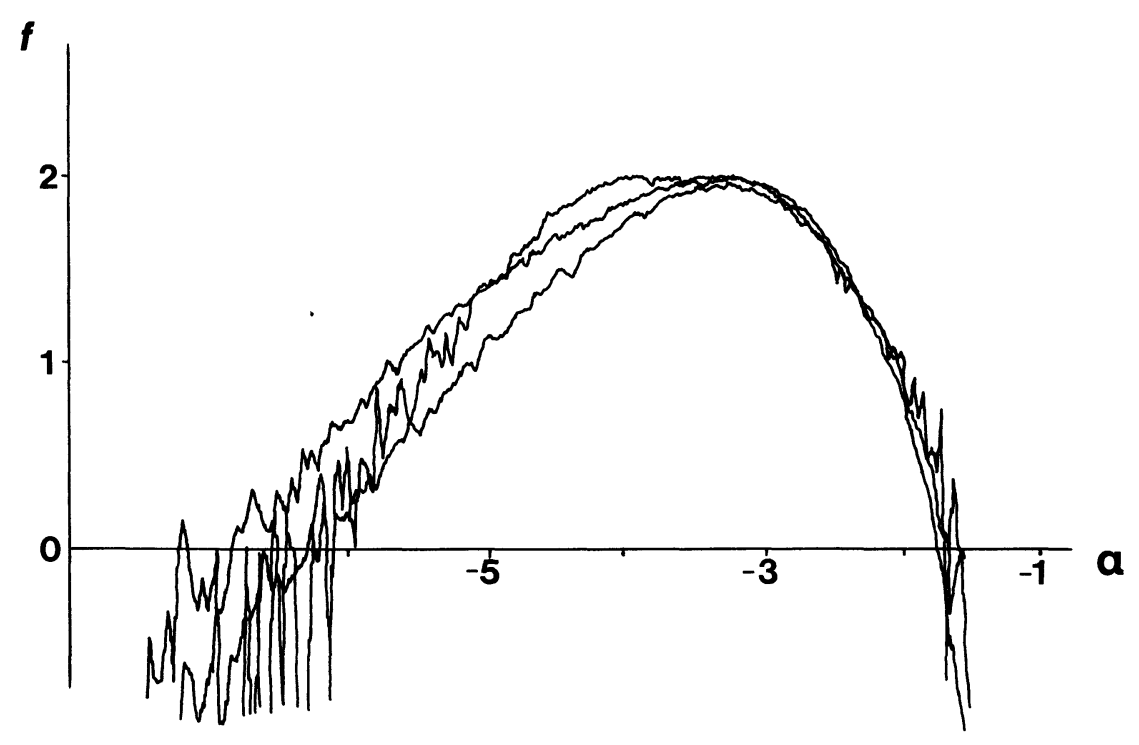

Fig. 7. - Rescaled log-histogram of the force distribution in the lattices just before the last bond breaks, for an imposed displacement of unity for three different sizes $L=8,16$ and 24 . This histogram should tend toward the multifractal spectrum « $f(\alpha)$ » when the lattice size increases (note that the $\alpha$ quoted here has nothing to do with the coefficient appearing in Eq. (5)). We observe a good collapse of the data, besides a small hump on the left of the apex of the curve that comes from the largest lattice size, where the statistics is poor.

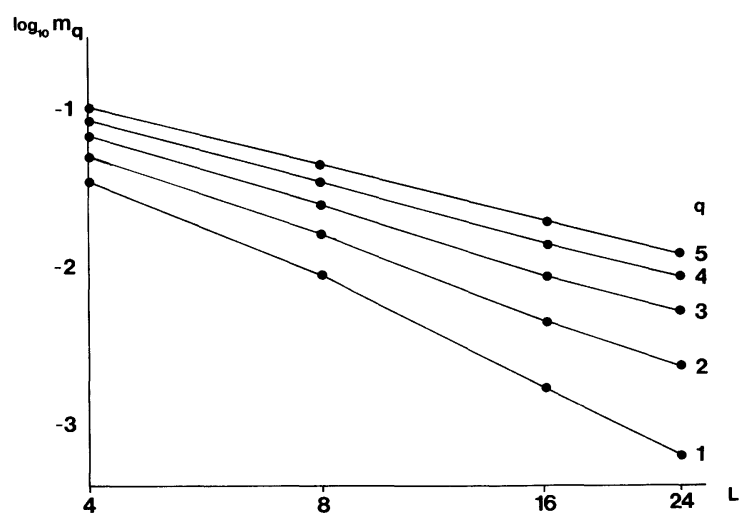

Fig. 8. - Evolution of the reduced moments $m_{q}$ of the force distribution just before the last bond breaks, versus the lattice size in a $\log$-log scale. The slope $y(q)$ of these moments from $q=1$ to 5 varies, and thus reveals a multifractal behavior.

$L$ for $q=1$ to 5 . The fact that these moments do not fall onto parallel lines in a log-log plot implies a multifractal distribution of forces, or a non constant gap scaling. From these plots, we can extract the difference slopes $y(q)$ and thus the corresponding exponent $p(q)=$ $p(0)-q y(q)$. Figure 9 gives the estimates of $p(q)$ that are also reported in table I. For high order of the moments we can approximate $p(q)$ by a linear relation $: p(q) \approx a q-b$. The 
Table I. - We report here the exponent $p(q)$ of the scaling of the moments of the force distribution : $p(0)$ is the negative of the fractal dimension of the force carrying part of the lattice thus -2 . $p(q)$ tends approximately to an asymptote $p(q) \approx 0.8 q-0.1$ for high order moments, revealing a localised zone of high stresses (of fractal dimension of order 0.1, eventually 0 ).
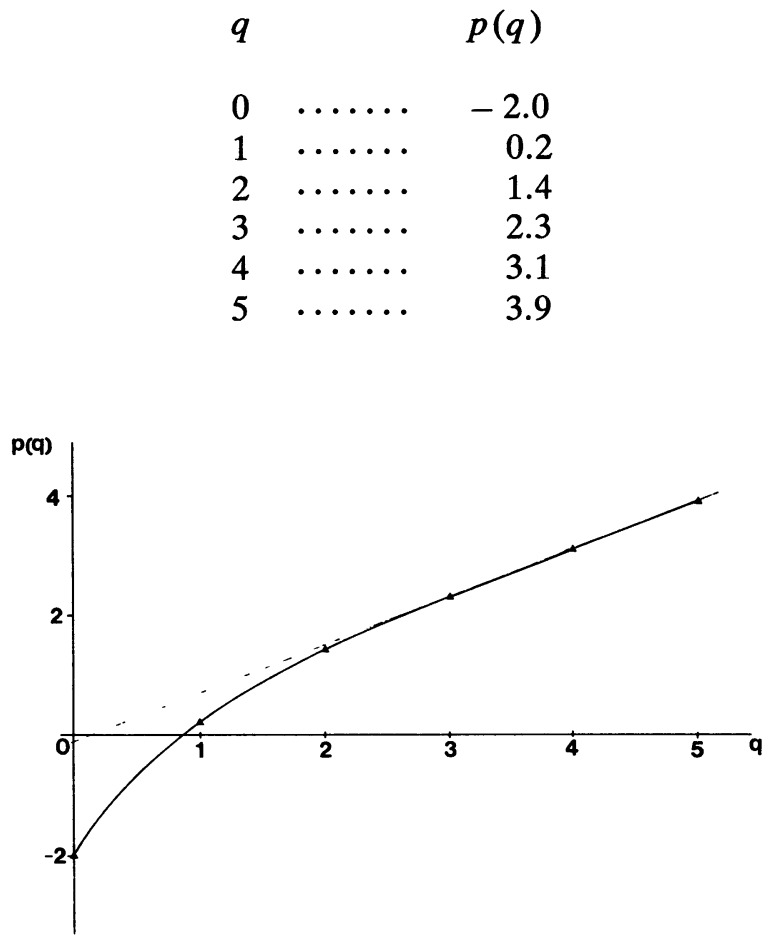

Fig. 9. - Evolution of the exponents $p(q)$ of the raw moments of the force distribution versus $q$. This curve shows again the multifractal distribution of forces.

physical meaning of the quantity $b$ is the fractal dimension of the subset of bonds that carry the highest forces. The numerical estimate of $b$ is 0.1 is consistent with the picture that just before the last bond breaks, the highest stresses are concentrated in a local zone, independent of the lattice size. Indeed, in this latter case, the dimension $b$ would be zero. To connect the series $p(q)$ to the multifractal spectrum « $f(\alpha)$, we recall the relations [16] :

$$
\begin{aligned}
& \alpha(q)=-\partial p(q) / \partial q \\
& f(\alpha)=f(\alpha(q))=-q \alpha(q)-p(q) .
\end{aligned}
$$

The occurrence of multifractality has been seen clearly in the fuse case of rupture $[7,8]$ at the final rupture point. However, the numerical values of $p(q)$ seem to be different in these two cases.

We now turn to the comparison of our results with those already reported [11] for the case of percolation-type disorder. This case can be cast in the same language as our model by recognizing that the percolation case can be viewed as a breaking strength distribution consisting in two Dirac functions, one situated at unity which a weight $p$ and the other at zero (i.e. the bonds break immediately) with a weight $(1-p)$ (see Fig. 10). Such a distribution leads to the following main results : 


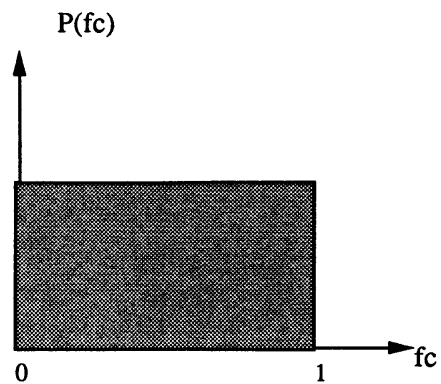

a)

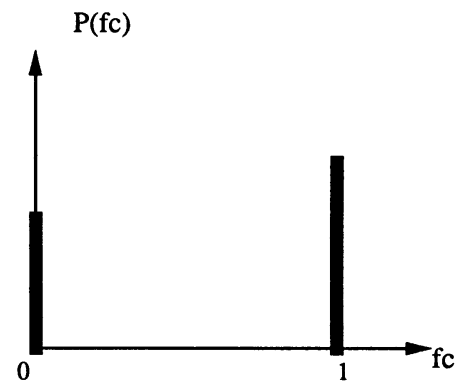

b)

Fig. 10. - Distribution of failure strength $f_{\mathrm{c}}$ used in our study (a) and in a percolation type disorder (b).

- the average initial breakdown force behaves as

$$
F \propto L(A(p)+B(p) \log (L))^{-1 / \mu}
$$

where $\mu$ is an exponent between 1 and 2 ;

- the global rupture force distribution has the following form :

$$
\varphi\left(F_{\max }\right) \propto \exp \left(-c L^{2} \exp \left(-k /\left(F_{\max } / L\right)^{\mu}\right)\right)
$$

from this last expression, Beale and Srolovitz deduced the average failure force as :

$$
F_{\max } \propto L\left(A^{\prime}(p)+B^{\prime}(p) \log (L)\right)^{-1 / \mu} .
$$

The first scaling relation (13) has no meaningful equivalent in the case of a continuous distribution of failure strength, such as the one studied in the present work. We did not study the global breakdown force distribution, but rather its average as discussed previously. The power-law dependence over the lattice size we have observed (Eq. (11)), is not consistent with the percolation type disorder results (Eq. (15)). The discrepancy between the results may be attributed to the existence of two peaks in the distribution of failure strength, separated by a gap for the percolation type disorder. Therefore in this case, the breaking is solely determined by the force distribution in the lattice. For the continuous distribution of local failure thresholds we considered, the rupture process proceeds continuously as a competition between the force and the failure strength distributions. This difference is the only possible origin of the different scaling laws observed.

\section{Conclusion.}

Let us summarize the main points obtained in this study.

- The relation between the breaking displacement and the number of bonds broken is remarkably linear over a substantial range (even beyond the apex of the breaking force).

- The relation between the breaking force and the number of bonds broken can be very well approximated by a parabolic law, in the first stages of the breaking process.

- The beginning of the force-displacement characteristics can be rescaled through a power-law dependence with an exponent of about $3 / 4$ on the lattice size, which seems very general, and non-trivial.

- The distribution of forces, just before the final completion of the rupture, is multifractal. 
These numerical observations certainly deserve confrontation with experimental data, as well as new theoretical approaches in order to understand, at least, the unexpected power-law dependence with size of most observable physical quantities on the system size.

\section{Acknowledgments.}

It is a pleasure to acknowledge useful discussions with L. de Arcangelis, E. Guyon and E. Hinrichsen. We are indebted to M. Novotny, and IBM Bergen Scientific Centre, for their hospitality in Bergen where most of the computations were performed. A.H. is supported by SFB 125 of the DFG, and S.R. by an ATP contract of the CNRS.

\section{References}

[1] See e.g. Fracture Vol. I-VII, Ed. H. Liebowitz (Acad. Press, N.Y.) 1984.

[2] De Arcangelis L., Redner S. and Herrmann H. J., J. Phys. Lett. France 46 (1985) L585.

[3] Duxbury P. M., Beale P. D. and Leath P. L., Phys. Rev. Lett. 57 (1986) 1052 ;

Duxbury P. M., Leath P. L. and Beale P. D., Phys. Rev. B 36 (1987) 1701.

[4] Sahimi M. and Goddard J., Phys. Rev. B 33 (1986) 7848 ;

Gilabert A., Vanneste C., Sornette D. and Guyon E., J. Phys. France 48 (1987) 763.

[5] Khang B., Batrouni G. G., Redner S., de Arcangelis L. and Herrmann H. J., Phys. Rev. B 37 (1988) 7625.

[6] Kantor Y. and Webman I., Phys. Rev. Lett. 52 (1984) 1891.

[7] De Arcangelis L., Hansen A., Herrmann H. J. and Roux S., preprint.

[8] Herrmann H. J., Hansen and Roux S., preprint.

[9] De Arcangelis L. and Herrmann H. J., preprint.

[10] Day A. R., Tremblay R. R., Tremblay A.-M. S., Phys. Rev. Lett. 56 (1986) 2501.

[11] Beale P. D. and Srolovitz D. J., Phys. Rev. B 37 (1988) 5500.

[12] Batrouni G. G., Hansen A. and Roux S., Phys. Rev. A 38 (1988) 3820.

[13] Batrouni G. G., Hansen A. and Nelkin M., Phys. Rev. Lett. 57 (1986) 1336 ;

Batrouni G. G. and Hansen A., J. Stat. Phys. 52 (1988) 747.

[14] Feng S., Thorpe M. F. and Garboczi E., Phys. Rev. B 31 (1985) 276.

[15] Roux S., Hansen A., Herrmann H. J. and Guyon E., J. Stat. Phys. 52 (1988) 237.

[16] Paladin G. and Vulpiani A., Phys. Rep. 156 (1987) 147 ;

De ARCANGelis L., Disorder and Mixing, Proc. of the Summer School Cargèse (June 14-27, 1987). 